Pacific Journal of Mathematic 


\title{
BURKILL-CESARI INTEGRALS OF QUASI ADDITIVE INTERVAL FUNCTIONS
}

\section{J. C. BRECKenRIDge}

\begin{abstract}
The Burkill-Cesari B-C integrals arising in L. Cesari's theory of quasi additive vector-valued set functions need not be additive as functions of sets. It is shown in the present paper that these integrals satisfy quasi additivity and overadditivity properties. These properties are used to prove Banach-type differentiation theorems for B-C integrals defined on Euclidean spaces. Variants of Cesari's basic quasi additivity hypothesis and some simplifications in the formulation of the general theory are also discussed.
\end{abstract}

The theory of quasi additive vector-valued interval functions $z$ and associated B-C integrals $\int[z, A]$ over an abstract space $A$ was introduced by Cesari [2]. The integration processes of Cauchy-Riemann and Lebesgue-Stieltjes were shown to be included in this theory. More importantly, it was proved that the property of quasi additivity is preserved by parametric integrands $f(p, q)$ and that CesariWeierstrass integrals over a variety $T$ are the B-C integrals $\int[f(T, z), A]$ of the corresponding composite functions. In [3] Cesari extended these concepts to subsets of $A$ and determined conditions under which the Cesari-Weierstrass integral can be represented as a Lebesgue integral $\int_{A} f(T, \theta) d \mu$ with respect to a suitable measure $\mu$ and vector $\theta$ of Radon-Nikodym derivatives. Further developments in the theory have been discussed by Nishiura [4], Stoddart [6], and Warner $[7,8,9]$. In particular, Warner extended the theory to include quasi additive functions with values in locally convex spaces and showed that many other integration processes, including those of Perron and Pettis, are contained in the theory.

In this paper we discuss properties of B-C integrals as functions defined on the subsets of a given space $A$. It is convenient to follow the original setting of Cesari. Thus the B-C integral $\int[z, M]$ of $z$ over an arbitrary subset $M$ of $A$ exists whenever $z$ is quasi additive on $M$. We differ from Cesari's procedure, however, by formulating all quasi additivity relations relative to a single directed system. This technical device, used by Nishiura [4] in surface area theory, simplifies Cesari's formulation and allows us to prove, in $\S 1$, that if $z$ is quasi additive on $M$ and if the B-C integral $\int[|z|, M]$ is finite, 
then $z$ is quasi additive on every subset $M^{\prime}$ of $M$; hence $\int\left[z, M^{\prime}\right]$ exists for every $M^{\prime} \subset M$.

Structure theorems for B-C integrals are discussed in $\$ 2$. In addition to the theorems already proved by Cesari, we prove that if $z$ is quasi additive on $M$ and $\int[|z|, M]$ is finite, then the interval function $\int[z, \cdot]$ is also quasi additive on $M$ and

$$
\int[z, M]=\int\left[\int[z, \cdot], M\right]
$$

if, in addition, $z$ is real valued and nonnegative, then $\int[z, M]$ is the total variation of $\int[z, \cdot]$ over $M$.

The structure theorems of $\S 2$ are used in $\S 3$ to prove Banachtype differentiation theorems for the case in which $A$ is an open set in Euclidean space. It is shown that if $z$ is quasi additive on $A$ and $\int[|z|, A]$ is finite, then the interval function $\int[z, \cdot]$ admits a "hard analysis" vector-valued derivative $J$ such that $\int[|z|, A] \geqq \int_{A}|J(w)| d w$; equality holds if and only if $\int[|z|, \cdot]$ is $A C$ in the sense of [1, p. 411]. This result, which we shall use in a later paper, is obtained by extracting essential elements in Cesari's presentation of the theory of generalized Jacobians associated with a parametric surface of finite area [1].

Stronger types of quasi additivity relations are discussed in $\S 4$. Necessary and sufficient conditions in terms of the interval function $\int[|z|, \cdot]$ are determined for the basic quasi additivity hypothesis to be equivalent to a stronger hypothesis used by Cesari [3, p. 130].

The connection between the present formulation and that of Cesari is discussed in $\S 5$. It is observed that Cesari's representation theorem, $\int[f(T, z), A]=\int_{A} f(T, \theta) d \mu$, holds in the present setting also.

1. Definitions and first properties. Let $A$ be a nonempty set, $\{I\}$ be a nonempty collection of subsets $I$ of $A,\{D\}$ be a nonempty family of nonempty finite systems $D=[I]$ of sets $I$ in $\{I\}$, and $\delta$ be a real-valued function defined on $\{D\}$. We refer to the sets in $\{I\}$ as intervals and to the function $\delta$ as a mesh. The axioms

(a): $A$ is a topological space,

(b): each interval $I$ has a nonempty interior, 
(c): the intervals of each system $D$ are nonoverlapping, i.e., int $(I) \cap \operatorname{cl}(J)=\operatorname{cl}(I) \cap \operatorname{int}(J)=\varnothing$ whenever $I, J \in D, I \neq J$,

(d): $0<\delta(D)<\infty$ for each system $D$ and, given $\varepsilon>0$, there are systems with $\delta(D)<\varepsilon$,

will be assumed. In order to treat some trivial examples in a uniform manner we allow $\delta(D)=0$ if only the zero (real or vectorvalued) interval function (see below) is being considered.

The norm of a point $q=\left(q_{1}, \cdots, q_{m}\right)$ in the real Euclidean $m$-space $E_{m}$ is denoted by $|q|=\left[\Sigma_{r} q_{r}^{2}\right]^{1 / 2}$, where $\Sigma_{r}$ ranges over $r=1, \cdots, m$. We set $a^{ \pm}=(|a| \pm a) / 2$ for any real number $a$.

Let $z:\{I\} \rightarrow E_{m}, z=\left(z_{1}, \cdots, z_{m}\right)$, be an interval function and $M$ be a subset of $A$. Associated with $z$ are the nonnegative interval functions $|z|,\left|z_{r}\right|, z_{r}^{+}$, and $z_{r}^{-}, r=1, \cdots, m$. When needed, $z^{\prime}$ denotes a second interval function having the same range space as $z$.

Given a system $D_{0}=[I]$, let $S\left[z, M, D_{0}\right]=\Sigma_{I} s(I, M) z(I)$, where $\Sigma_{I}$ ranges over all $I \in D_{0}$ and $s(I, M)=1$ or 0 according as $I \subset M$ or $I \not \subset M$. If $D=[J]$ is also a system, then $S[z, M, D]-S\left[z, M, D_{0}\right]=$ $\Sigma_{I} s(I, M)\left[\Sigma_{J} s(J, I) z(J)-z(I)\right]+\Sigma_{J} s(J, M)\left[1-\Sigma_{I} s(J, I) s(I, M)\right] z(J)$; the second term on the right is nonnegative whenever $z$ is nonnegative.

The $B$-C integral of $z$ over $M$ is defined as

$$
\int[z, M]=\lim S[z, M, D]
$$

provided this limit, taken as $\delta(D) \rightarrow 0$, exists in $E_{m}$. If $z$ is realvalued, then $\infty$ is also allowed as a value for this integral.

Definition 1.1. $z$ is quasi additive on $M$ if for each $\varepsilon>0$ there exists $\eta=\eta(\varepsilon, M)>0$ such that if $D_{0}=[I]$ is any system satisfying $\delta\left(D_{0}\right)<\eta$, then there also exists $\lambda=\lambda\left(\varepsilon, M, D_{0}\right)>0$ such that the relations

$\left(q a_{1}\right): \quad \Sigma_{I} s(I, M)\left|\Sigma_{J} s(J, I) z(J)-z(I)\right|<\varepsilon$,

$\left(q a_{2}\right): \quad \Sigma_{J} s(J, M)\left[1-\Sigma_{I} s(J, I) s(I, M)\right]|z(J)|<\varepsilon$,

hold for every system $D=[J]$ with $\delta(D)<\lambda$. If $z$ is real-valued and if $\left(q \alpha_{1}\right)$ and $\left(q a_{2}\right)$ are replaced by the single relation

$(q s a): \quad \Sigma_{I} s(I, M)\left[\Sigma_{J} s(J, I) z(J)-z(I)\right]^{-}<\varepsilon$, then $z$ is quasi subadditive on $M$.

Proposition 1.2. (i) If $z$ is quasi additive on $M$, then the $B-C$ integral $\int[z, M]$ exists in $E_{m}$.

(ii) If $z$ is nonnegative and quasi subadditive on $M$, then $\int[z, M]$ exists, $0 \leqq \int[z, M] \leqq \infty$. If, in addition, this B-C integral is finite, 
then $z$ is quasi additive on $M$.

(iii) If $z$ and $z^{\prime}$ are quasi additive on $M$, then $a z+b z^{\prime}$ is quasi additive on $M$ for every pair of real numbers $a$ and $b$, and

$$
\int\left[a z+b z^{\prime}, M\right]=a \int[z, M]+b \int\left[z^{\prime}, M\right] \text {. }
$$

(iv) If $z$ and $z^{\prime}$ are nonnegative and quasi subadditive on $M$ and $M^{\prime}$, respectively, and if $z^{\prime} \leqq z, M^{\prime} \subset M$, then $\int\left[z^{\prime}, M^{\prime}\right] \leqq \int[z, M]$.

(v) $z$ is quasi additive on $M$ if and only if its components $z_{r}$, $r=1, \cdots, m$, are all quasi additive on $M$.

(vi) If $z$ is quasi additive on $M$, then $|z|,\left|z_{r}\right|, z_{r}^{+}$, and $z_{r}^{-}$, $r=1, \cdots, m$, are all quasi subadditive on $M$.

The proofs of the preceding statements are analogous to the proofs given in [2, pp. 97-99] for the case $M=A$.

If $z$ is quasi additive on $M$ and if there exist systems of arbitrarily small mesh, none of whose intervals are contained in $M$, then $\int[z, M]$ is the zero vector. In particular, $z$ is automatically quasi additive on the empty set and $\int[z, \varnothing]$ is the zero vector.

It follows from relation $(q s a)$ that if $\mathrm{z}$ is quasi subadditive on $M$, then it is quasi subadditive on every subset of $M$.

Proposition 1.3. If $z$ is quasi additive on $M$ and if $\int[|z|, M]$ is finite, then $z$ is quasi additive on every subset of $M$.

Proof. Let $M^{\prime} \subset M$ be given. We shall refer to the statements of (1.2). By (vi), $|z|$ is quasi subadditive on $M$ and therefore also on $M^{\prime}$. By (ii) and (iv), $\int\left[|z|, M^{\prime}\right]$ exists and is dominated by $\int[|z|, M]<\infty$. Thus $|z|$ is quasi additive on $M^{\prime}$ by (ii). Given $\varepsilon>0$ we can determine the parameters of (1.1) so that the relations $\left(q a_{1}\right)$ and $\left(q a_{2}\right)$ are simultaneously satisfied relative to $z$ on $M$ and $|z|$ on $M^{\prime}$. Thus

$$
\begin{aligned}
& \Sigma_{I} s\left(I, M^{\prime}\right)\left|\Sigma_{J} s(J, I) z(J)-z(I)\right| \\
\leqq & \Sigma_{I} s(I, M)\left|\Sigma_{J} s(J, I) z(J)-z(I)\right|<\varepsilon, \\
& \Sigma_{J} s\left(J, M^{\prime}\right)\left[1-\Sigma_{I} s(J, I) s\left(I, M^{\prime}\right)\right]|z(J)|<\varepsilon,
\end{aligned}
$$

and we conclude that $z$ is quasi additive on $M^{\prime}$.

It is convenient to set 


$$
\begin{gathered}
F(M)=\int[|z|, M], \quad F_{r}(M)=\int\left[\left|z_{r}\right|, M\right], \\
F_{r}^{+}(M)=\int\left[z_{r}^{+}, M\right], \quad F_{r}^{-}(M)=\int\left[z_{r}^{-}, M\right], \\
\mathscr{F}_{r}(M)=\int\left[z_{r}, M\right],
\end{gathered}
$$

whenever these B-C integrals exist and are finite, and to set $F(M)=0$, etc., otherwise. We also define

$$
\mathscr{F}(M)=\left(\mathscr{F}_{1}(M), \cdots, \mathscr{F}_{m}(M)\right) \text {. }
$$

As a consequence of the definitions and preceding propositions we have the following result.

Proposition 1.4. Suppose that $z$ is quasi additive on $M$ and that $\int[|z|, M]$ is finite. Let $M^{\prime} \subset M$ be given. Then $z, z_{r},|z|,\left|z_{r}\right|$, $z_{r}^{+}$, and $z_{r}^{-}, r=1, \cdots, m$, are all quasi additive on $M^{\prime}$, and

$$
\begin{gathered}
F_{r}^{+}\left(M^{\prime}\right)-F_{r}^{-}\left(M^{\prime}\right)=\mathscr{F}_{r}\left(M^{\prime}\right), \\
F_{r}^{+}\left(M^{\prime}\right)+F_{r}^{-}\left(M^{\prime}\right)=F_{r}\left(M^{\prime}\right), \\
\left|\mathscr{F}_{r}\left(M^{\prime}\right)\right| \leqq F_{r}\left(M^{\prime}\right) \leqq F\left(M^{\prime}\right), \\
\left|\mathscr{F}^{\prime}\left(M^{\prime}\right)\right| \leqq\left[\Sigma_{r} F_{r}^{2}\left(M^{\prime}\right)\right]^{1 / 2} \leqq F\left(M^{\prime}\right) \leqq \Sigma_{r} F_{r}\left(M^{\prime}\right),
\end{gathered}
$$

for each $r$. Given $\varepsilon>0$, there exists $\mu=\mu\left(\varepsilon, M^{\prime}\right), 0<\mu \leqq \varepsilon$, such that if $D_{0}=[I]$ is any system with $\delta\left(D_{0}\right)<\mu$, then

$$
\left|\mathscr{F}\left(M^{\prime}\right)-S\left[z, M^{\prime}, D_{0}\right]\right|<\varepsilon,\left|F\left(M^{\prime}\right)-S\left[|z|, M^{\prime}, D_{0}\right]\right|<\varepsilon,
$$

and analogously for $\mathscr{F}_{r}, F_{r}, F_{r}^{+}$and $F_{r}^{-}$for each $r$. Finally, there exists $\lambda=\lambda\left(\varepsilon, M^{\prime}, D_{0}\right), 0<\lambda \leqq \mu$, such that the relations $\left(q a_{1}\right)$ and $\left(q a_{2}\right)$ of (1.1) (applied to $M^{\prime}$ ) hold simultaneously for $z, z_{r},|z|,\left|z_{r}\right|, z_{r}^{+}$, and $z_{r}^{-}$for every $r$ and every system $D=[J]$ with $\delta(D)<\lambda$.

2. B-C integrals as interval functions. The total variation (relative to $\{D\}$ ) of $z$ over $M$ is defined as

$$
V[z, M]=\sup S[|z|, M, D]
$$

where the supremum is taken over all systems $D \in\{D\}$. We have $0=V[z, \varnothing] \leqq V\left[z, M^{\prime}\right] \leqq V[z, M] \leqq V[z, A] \leqq \infty$ whenever $M^{\prime} \subset M$. If $z$ is quasi additive on $M$, then $\int[|z|, M] \leqq V[z, M]$ and strict inequality may hold.

Proposition 2.1. If $\{D\}$ is the family of all nonempty finite systems of nonoverlapping intervals $I \in\{I\}$, then 
(i) $V[z, M] \geqq \sum_{n=1}^{\infty} V\left[z, M_{n}\right]$ for every sequence $\left\{M_{n}\right\}$ of nonoverlapping subsets $M_{n}$ of $M$,

(ii) if each interval $I \in\{I\}$ is connected, then

$$
V[z, G]=\sum_{n=1}^{\infty} V\left[z, G_{n}\right]
$$

whenever $\left\{G_{n}\right\}$ is a sequence of disjoint open subsets of $A$ such that $G=\cup_{n=1}^{\infty} G_{n}$.

These two properties of the total variation are well-known (cf. $[1,9.3]$ ). The connectedness of the intervals is assumed in (ii) to assure that if $I \subset G$, then $I \subset G_{n}$ for one and only one value of $n$.

In the next two results we assume that $z$ is nonnegative (realvalued). In this case we have $\mathscr{F}=F$.

THEOREM 2.2. If $z$ is nonnegative and quasi additive on $M$, then the interval function $F$ is also quasi additive on $M$, and

$$
F\left(M^{\prime}\right)=V\left[F, M^{\prime}\right]=\int\left[F, M^{\prime}\right]
$$

for every subset $M^{\prime}$ of $M$.

Proof. In view of (1.3), it suffices to take $M^{\prime}=M$. We first prove the equalities in (1). For any two systems $D_{0}=[I]$ and $D=[J]$ we have

$$
\begin{aligned}
0 & \leqq \sum_{J} s(J, M)\left[1-\sum_{I} s(J, I) s(I, M)\right] z(J) \\
& =\sum_{J} s(J, M) z(J)-\sum_{J} s(J, M) \sum_{I} s(J, I) s(I, M) z(J) \\
& =S[z, M, D]-\sum_{I} s(I, M) \sum_{J} s(J, I) z(J) \\
& =S[z, M, D]-\sum_{I} s(I, M) S[z, I, D]
\end{aligned}
$$

As $\delta(D) \rightarrow 0$ we obtain

$$
0 \leqq F(M)-\sum_{I} s(I, M) F(I)=F(M)-S\left[F, M, D_{0}\right] .
$$

Thus

$$
F(M) \geqq V[F, M] \geqq S\left[F, M, D_{0}\right]
$$

for every system $D_{0}$. If $\varepsilon>0$ and if $D_{0}$ and $D$ are as in (1.1), then we also have $0 \leqq S[z, M, D]-\sum_{I} s(I, M) S[z, I, D]<\varepsilon$. As $\delta(D) \rightarrow 0$ we thus obtain

$$
0 \leqq F(M)-S\left[F, M, D_{0}\right] \leqq \varepsilon
$$

for all systems $D_{0}$ of sufficiently small mesh. The equalities (1) follow 
from (2) and (3) since $\varepsilon>0$ was arbitrary.

It remains to show that $F$ is quasi additive on $M$. Let $\varepsilon>0$ be given and let $D_{0}=[I]$ be a system. If $\int[z, M]=0$, then the problem is trivial. We thus assume that $\int[z, M]>0$ and that $\delta\left(D_{0}\right)$ is small enough that the set $M$ contains at least one interval $I \in D_{0}$. Let $N$ be the number of intervals $I \in D_{0}$ with $I \subset M$. By (1) there exists $\lambda=\lambda\left(\varepsilon, M, D_{0}\right)>0$ such that

$$
0 \leqq V[F, I]-S[F, I, D]<\varepsilon / N
$$

for every system $D=[J]$ with $\delta(D)<\lambda$ and for every interval $I \in D_{0}$ with $I \subset M$. Since $V[F, I]=F(I)$ for each of these intervals, we have

$$
\sum_{I} s(I, M)\left[\sum_{J} s(J, I) F(J)-F(I)\right]^{-}<N(\varepsilon / N)=\varepsilon
$$

by (4). This proves that $F$ is quasi subadditive on $M$. By (1) we have $F(M)=\int[F, M]$ and this $\mathrm{B}-\mathrm{C}$ integral is finite. Thus $F$ is quasi additive on $M$ by statement (ii) of (1.2).

Proposition 2.3. If $z$ is nonnegative and quasi additive on $M$, then $F(M) \geqq \sum_{n=1}^{\infty} F\left(M_{n}\right)$ for every sequence $\left\{M_{n}\right\}$ of nonoverlapping subsets $M_{n}$ of $M$.

Proof. Let $\left\{D^{\prime}\right\}$ be the family of all nonempty finite systems of nonoverlapping intervals $I \in\{I\}$ and let $\delta^{\prime}$ be the mesh on $\left\{D^{\prime}\right\}$ defined by $\delta^{\prime}\left(D^{\prime}\right)=\delta\left(D^{\prime}\right)$ if $D^{\prime} \in\{D\}$ and $\delta^{\prime}\left(D^{\prime}\right)=1$ if $D^{\prime} \in\left\{D^{\prime}\right\}-\{D\} . \quad z$ is obviously quasi additive on $M$ relative to $\left(\left\{D^{\prime}\right\}, \delta^{\prime}\right)$, and $F\left(M^{\prime}\right)=\lim$ $S\left[z, M^{\prime}, D^{\prime}\right]$ as $\delta^{\prime}\left(D^{\prime}\right) \rightarrow 0$ for every set $M^{\prime} \subset M$. The proposition is now a consequence of (2.1) and (2.2) applied to $\left(\left\{D^{\prime}\right\}, \delta^{\prime}\right)$.

Examples (see [1, p. 400]) show that strict inequality may hold in the above proposition even if $M$ is the union of extensively overlapping sets $M_{n}$.

We now return to the case in which $z=\left(z_{1}, \cdots, z_{m}\right)$ is vectorvalued.

Proposition 2.4. Assume that each interval $I \in\{I\}$ is connected. Let $\left\{G_{n}\right\}$ be a sequence of disjoint open subsets of $A$ and let $G=\cup_{n=1}^{\infty} G_{n}$. If $z$ is quasi additive on $G$ and if $\int[|z|, G]$ is finite, then $\mathscr{F}(G)=\sum_{n=1}^{\infty} \mathscr{F}\left(G_{n}\right)$ and the series is absolutely convergent. Analogous statements hold for $\mathscr{F}_{r}, F, F_{r}, F_{r}^{+}$, and $F_{r}^{-}, r=1, \cdots, m$. 
Proof. As shown in the proof of the preceding proposition, it is not restrictive to assume that $\{D\}$ is the family of all finite systems of nonoverlapping intervals. The desired equalities thus hold for $F, F_{r}, F_{r}^{+}$, and $F_{r}^{-}$by $(2.1)$ and (2.2). These equalities extend to $\mathscr{F}_{r}$ and $\mathscr{F}$ by virtue of the relations $\mathscr{F}_{r}=F_{r}^{+}-F_{r}^{-}$and $\mathscr{F}=$ $\left(\mathscr{F}_{1}, \cdots, \mathscr{F}_{m}\right)$. Absolute convergence for the latter series holds since $\left|\mathscr{F}_{r}\right| \leqq F_{r}$ and $|\mathscr{F}| \leqq F$.

Another proof of (2.4) has been given under slightly different hypotheses by Cesari [3, p. 118].

THEOREM 2.5. If $z$ is quasi additive on $M$ and if $\int[|z|, M]$ is finite, then the interval function $\mathscr{F}$ is quasi additive on $M$ and $\mathscr{F}\left(M^{\prime}\right)=\int\left[\mathscr{F}, M^{\prime}\right]$ for every subset $M^{\prime}$ of $M$.

Proof. The interval functions $F_{r}^{+}$and $F_{r}^{-}, r=1, \cdots, m$, are quasi additive on $M$ by (2.2). The functions $\mathscr{F}_{r}=F_{r}^{+}-F_{r}^{-}$are thus quasi additive on $M$ by (1.2) (iii). Hence $\mathscr{F}$ is quasi additive on $M$ by (1.2) (v). From (1.4) and (2.2) we have

$$
\mathscr{F}_{r}\left(M^{\prime}\right)=F_{r}^{+}\left(M^{\prime}\right)-F_{r}^{-}\left(M^{\prime}\right)=\int\left[F_{r}^{+}, M^{\prime}\right]-\int\left[F_{r}^{-}, M^{\prime}\right]=\int\left[\mathscr{F}_{r}, M^{\prime}\right]
$$

and we conclude that $\mathscr{F}\left(M^{\prime}\right)=\int\left[\mathscr{F}, M^{\prime}\right]$ for every subset $M^{\prime}$ of $M$.

THEOREM 2.6. If $z$ is quasi additive on $M$ and if $\int[|z|, M]$ is finite, then

$$
F\left(M^{\prime}\right)=\lim _{\delta\left(D_{0}\right) \rightarrow 0} \sum_{I} s\left(I, M^{\prime}\right)\left[\sum_{r} F_{r}^{2}(I)\right]^{1 / 2}
$$

for every subset $M^{\prime}$ of $M$. Here, $D_{0}=[I] \in\{D\}$.

Proof. By (1.3) it suffices to take $M^{\prime}=M$. Let $\varepsilon>0$ be given. Let $\mu=\mu(\varepsilon, M)$ with $0<\mu \leqq \varepsilon, D_{0}=[I]$ with $\delta\left(D_{0}\right)<\mu, \lambda=\lambda\left(\varepsilon, M, D_{0}\right)$ with $0<\lambda \leqq \mu$, and $D=[J]$ with $\delta(D)<\lambda$ be as in (1.4). Then

$$
\begin{gathered}
\left|F(M)-\sum_{I} s(I, M)\right| z(J)|-| z_{r}(I)||<\varepsilon, \\
\sum_{I} s(I, M)\left|\sum_{J} s(J, I)\right| z_{r}(J)|-| z_{r}(I)||<\varepsilon,
\end{gathered}
$$

for each $r=1, \cdots, m$. For each $r$ and $I \in D_{0}$, let

$$
a_{r}(I)=\sum_{J} s(J, I)\left|z_{r}(J)\right|-\left|z_{r}(I)\right| \text {. }
$$

By substitution and Minkowski's inequality we obtain 


$$
\begin{aligned}
F(M) & <\sum_{I} s(I, M)|z(I)|+\varepsilon \\
= & \sum_{I} s(I, M)\left\{\sum_{r}\left|z_{r}(I)\right|^{2}\right\}^{1 / 2}+\varepsilon \\
= & \sum_{I} s(I, M)\left\{\sum_{r}\left[\sum_{J} s(J, I)\left|z_{r}(J)\right|-a_{r}(I)\right]^{2}\right\}^{1 / 2}+\varepsilon \\
\leqq & \sum_{I} s(I, M)\left\{\sum_{r}\left[\sum_{J} s(J, I)\left|z_{r}(J)\right|+\left|a_{r}(I)\right|\right]^{2}\right\}^{1 / 2}+\varepsilon \\
\leqq & \sum_{I} s(I, M)\left\{\sum_{r}\left[\sum_{J} s(J, I)\left|z_{r}(J)\right|\right]^{2}\right\}^{1 / 2} \\
& +\sum_{I} s(I, M)\left\{\sum_{r}\left|a_{r}(I)\right|^{2}\right\}^{1 / 2}+\varepsilon \\
\leqq & \sum_{I} s(I, M)\left\{\sum_{r}\left[\sum_{J} s(J, I)\left|z_{r}(J)\right|\right]^{2}\right\}^{1 / 2} \\
& +\sum_{r} \sum_{I} s(I, M)\left|a_{r}(I)\right|+\varepsilon \\
& <\sum_{I} s(I, M)\left\{\sum_{r}\left[\sum_{J} s(J, I)\left|z_{r}(J)\right|\right]^{2}\right\}^{1 / 2}+(m+1) \varepsilon .
\end{aligned}
$$

As $\delta(D) \rightarrow 0$ and with the help of (2.2) we obtain

$$
F(M) \leqq \sum_{I} s(I, M)\left[\sum_{r} F_{r}^{2}(I)\right]^{1 / 2}+(m+1) \varepsilon
$$

for all systems $D_{0}=[I]$ with $\partial\left(D_{0}\right)<\mu(\varepsilon, M) \leqq \varepsilon$. As $\varepsilon>0$ was arbitrary, we have

$$
F(M) \leqq \underline{\lim }_{\hat{\delta}\left(D_{\jmath}\right) \rightarrow 0} \sum_{I} s(I, M)\left[\sum_{r} F_{r}^{2}(I)\right]^{1 / 2}, D_{0}=[I] .
$$

By (2.2) and (1.4), on the other hand, we have

$$
F(M) \geqq \sum_{I} s(I, M) F(I) \geqq \sum_{I} s(I, M)\left[\sum_{r} F_{r}^{2}(I)\right]^{1 / 2}
$$

for every system $D_{0}=[I]$. This competes the proof.

Note that we have also proved

$$
F(M)=\sup \sum_{I} s(I, M)\left[\sum_{r} F_{r}^{2}(I)\right]^{1 / 2}
$$

where the supremum is taken over all systems $D_{0}=[I]$.

3. Derivatives. Points of $E_{k}, k \geqq 1$, will be denoted by $w=\left(w_{1}, \cdots, w_{k}\right)$. The interior and frontier of a set $E$ in $E_{k}$ will be denoted by $E^{\circ}$ and $E^{*}$, respectively. The term a.e. (almost everywhere) will be used relative to $k$-dimensional Lebesgue measure $L_{k}$ on $E_{k}$.

Throughout this section A will denote a nonempty open subset of $E_{k}$ and $\{I\}$ the collection of all nondegenerate closed intervals $I=\left\{w \in E_{k}: a_{i} \leqq w_{i} \leqq b_{i}, i=1, \cdots, k\right\}$ contained in $A$. $\{D\}$ will denote the family of all non-empty finite systems $D=[I]$ of nonoverlapping sets $I \in\{I\}$. We assume that a mesh $\delta$ on $\{D\}$ is given. The definitions and results of this section may also be used if $\{I\}$ is replaced by the collection of all polyhedral regions or simple polyhedral regions (see [4]) contained in $A$.

We recall some definitions. A real-valued interval function $z$ is said to be

(i ) overadditive if $z(I) \geqq \sum_{j} z\left(I_{j}\right)$ for each set $I \in\{I\}$ and each 
finite subdivision $I=\cup_{j} I_{j}$ of $I$ into nonoverlapping sets $I_{j} \in\{I\}$,

(ii) additive if equality holds in (i),

(iii) $B V$ if the total variation $V[z, A]$ (relative to $\{D\}$ ) is finite,

(iv) $A C$ if (a) $z$ is additive, and (b) for each $\varepsilon>0$ there exists $\eta>0$ such that $S[|z|, A, D]<\varepsilon$ for each system $D$ with $S\left[L_{k}, A, D\right]<\eta$. Conditions (a) and (b) in (iv) are independent [1, p. 216].

Given $w \in A$, let $Q$ be generic for a closed $k$-cube, $Q \subset A$, with faces parallel to the coordinate hyperplanes of $E_{k}$ and with $w \in Q^{\circ}$. The derivative of a real-valued interval function $z$ at the point $w$ is defined as

$$
D(w, z)=\lim z(Q) / L_{k}(Q)
$$

provided this limit, taken as $L_{k}(Q) \rightarrow 0$, exists and is finite; otherwise we set $D(w, z)=0$. For the following theorem, see [1, Section 27] and [5, Section III. 2].

THEOREM 3.1. Suppose $z$ is nonnegative, overadditive, and $B V$. Then the limit (1) exists and is finite a.e. in $A, D(w, z)$ is Borel measurable and L-integrable on $A$, and $V[z, A] \geqq \int_{A} D(w, z) d w$. The same inequality holds if $A$ is replaced by any open set $G \subset A$ or by any set $I \in\{I\}$. The equality $V[z, A]=\int_{A} D(w, z) d w$ holds if and only if $z$ is $A C$ and, in this case, the same equality holds if $A$ is replaced by any $G$ or $I$ as above.

Let $z=\left(z_{1}, \cdots, z_{m}\right)$ be vector-valued. If $z$ is quasi additive on $A$ and if $\int[|z|, A]$ is finite, then the nonnegative interval functions $F, F_{r}, F_{r}^{+}$, and $F_{r}^{-}, r=1, \cdots, m$, are overadditive and $B V$ by (2.2) and (2.3). It is convenient to use the notations

$$
\begin{aligned}
D(w) & =D(w, F), & D_{r}(w) & =D\left(w, F_{r}\right), \\
D_{r}^{+}(w) & =D\left(w, F_{r}^{+}\right), & D_{r}^{-}(w) & =D\left(w, F_{r}^{-}\right), \\
J_{r}(w) & =D_{r}^{+}(w)-D_{r}^{-}(w), & J(w) & =\left(J_{1}(w), \cdots, J_{m}(w)\right) .
\end{aligned}
$$

From (1.4) we obtain $\left|J_{r}\right|=\left|D_{r}^{+}-D_{r}^{-}\right| \leqq D_{r}^{+}+D_{r}^{-}=D_{r} \leqq D$ a.e. in $A$ for each $r$.

In the next two results we assume that $z$ is real-valued.

Proposition 3.2. If $z$ is real-valued and quasi additive on $A$ and if $\int[|z|, A]$ is finite, then $D^{+}(w) D^{-}(w)=0$ and $|J(w)|=D(w)$ a.e. in $A$. 
Proof. Let $M_{1}=\left\{w \in A: D^{+}(w)>0\right\}$ and $M_{2}=\left\{w \in A: D^{-}(w)>0\right\}$. For each integer $n=1,2, \cdots$, let $M_{1 n}=\left\{w \in A: D^{+}(w)>1 / n\right\}$ and $M_{2 n}=\left\{w \in A: D^{-}(w)>1 / n\right\}$.

Let $\varepsilon>0$ and $n$ be given. Let $\mu=\mu(\varepsilon / 2 n, A)$ be as in (1.4) and let $D_{0}=[I]$ satisfy $\delta\left(D_{0}\right)<\mu$. Let $A_{1}$ be the union of all $I \in D_{0}$ such that $z^{+}(I)>0$, and let $A_{2}$ be the union of all $I \in D_{0}$ such that $z^{-}(I)>0$. Then the sets $G_{1}=A-A_{1}$ and $G_{2}=A-A_{2}$ are open. We show first that $L_{k}\left(G_{1} \cap M_{1 n}\right) \leqq \varepsilon$ and that $L_{k}\left(G_{2} \cap M_{2 n}\right) \leqq \varepsilon$.

Let $\lambda=\lambda\left(\varepsilon / 2 n, A, D_{0}\right)$ with $0<\lambda \leqq \mu$ be as in (1.4) and let $D=[J]$ be any system with $\delta(D)<\lambda$. Let $H_{1}$ be the closure of $G_{1}$ in $A$. Thus $G_{1} \subset H_{1} \subset A$ and $z^{+}(I)=0$ for every interval $I \in D_{0}$ with $I \subset H_{1}$. Also, if $J \in D, J \subset H_{1}$, and $J \not \subset I$ for any $I \in D_{0}$ with $I \subset H_{1}$, then $J \not \subset I$ for any $I \in D_{0}$. Hence,

$$
\begin{aligned}
0 \leqq & S\left[z^{+}, G_{1}, D\right] \leqq S\left[z^{+}, H_{1}, D\right]=\sum_{J} s\left(J, H_{1}\right) z^{+}(J) \\
= & \sum_{I} s\left(I, H_{1}\right)\left[\sum_{J} s(J, I) z^{+}(J)-z^{+}(I)\right] \\
& +\sum_{J} s\left(J, H_{1}\right)\left[1-\sum_{I} s(J, I) s\left(I, H_{1}\right)\right] z^{+}(J) \\
\leqq & \sum_{I} s(I, A)\left|\sum_{J} s(J, I) z^{+}(J)-z^{+}(I)\right| \\
& +\sum_{J} s(J, A)\left[1-\sum_{I} s(J, I) s(I, A)\right] z^{+}(J) \\
& <\varepsilon / 2 n+\varepsilon / 2 n=\varepsilon / n
\end{aligned}
$$

and, as $\partial(D) \rightarrow 0$, we obtain $0 \leqq F^{+}\left(G_{1}\right) \leqq \varepsilon / n$. From (2.2) and (3.1) we now have

$$
\begin{aligned}
0 & \leqq L_{k}\left(G_{1} \cap M_{1 n}\right) \leqq n \int_{G_{1}} D^{+}(w) d w \leqq n V\left[F^{+}, G_{1}\right] \\
& =n F^{\circ}\left(G_{1}\right) \leqq \varepsilon .
\end{aligned}
$$

An analogous argument shows that $L_{k}\left(G_{2} \cap M_{2 n}\right) \leqq \varepsilon$.

Since $A \subset\left[G_{1} \cup G_{2} \cup\left(A_{1}^{*} \cap A_{2}^{*}\right)\right]$ and $L_{l k}\left(A_{1}^{*} \cap A_{2}^{*}\right)=0$, we have

$$
\begin{aligned}
L_{k}\left(M_{1 n} \cap M_{2 n}\right)= & L_{k}\left(M_{1 n} \cap M_{2 n} \cap A\right) \\
\leqq & L_{k}\left[M_{2 n} \cap\left(M_{1 n} \cap G_{1}\right)\right]+L_{k}\left[M_{1 n} \cap\left(M_{2 n} \cap G_{2}\right)\right] \\
& +L_{k}\left(M_{1 n} \cap M_{2 n} \cap A_{1}^{*} \cap A_{2}^{*}\right) \\
\leqq & L_{k}\left(M_{1 n} \cap G_{1}\right)+L_{k}\left(M_{2 n} \cap G_{2}\right)<2 \varepsilon .
\end{aligned}
$$

As $\varepsilon>0$ and $n$ were arbitrary, we conclude that $L_{k}\left(M_{1 n} \cap M_{2 n}\right)=0$ for each $n$. From the construction of the sets $M_{1 n}$ and $M_{2 n}$ we conclude further that $L_{k}\left(M_{1} \cap M_{2}\right)=0$. Therefore $D^{+} D^{-}=0$ a.e. in $A$ and $|J|=\left|D^{+}-D^{-}\right|=D^{+}+D^{-}=D$ a.e. in $A$. This completes the proof.

Theorem 3.3. Suppose that $z$ is real-valued and quasi additive on $A$ and that $\int[|z|, A]$ is finite. Then 


$$
\begin{gathered}
F(A) \geqq \int_{A}|J(w)| d w, \quad F^{+}(A) \geqq \int_{A} D^{+}(w) d w, \\
F^{-}(A) \geqq \int_{A} D^{-}(w) d w,
\end{gathered}
$$

and the same inequalities hold if $A$ is replaced by any open subset $G$ of $A$ or by any set $I \in\{I\}$. The equality $F(A)=\int_{A}|J(w)| d w$ holds if and only if $F$ is $A C$. If $F$ is $A C$, then

$$
\begin{aligned}
F(A) & =\int_{A}|J(w)| d w, & & F^{+}(A)=\int_{A} D^{+}(w) d w, \\
F^{-}(A) & =\int_{A} D^{-}(w) d w, & \mathscr{F}(A) & =\int_{A} J(w) d w,
\end{aligned}
$$

and the same equalities hold if $A$ is replaced by any $G$ or $I$ as above.

Proof. Recalling that $F, F^{+}$, and $F^{-}$are nonnegative and overadditive and that $F=F^{+}+F^{-}$, it is easily verified that $F$ is $A C$ if and only if $F^{+}$and $F^{-}$are both $A C$. The theorem now follows from (2.2), (3.1), (3.2), and the relation $\mathscr{F}=F^{+}-F^{-}$.

We return now to the case in which $z=\left(z_{1}, \cdots, z_{m}\right)$ is vectorvalued.

Proposition 3.4. Suppose that $z$ is quasi additive on $A$ and that $\int[|z|, A]$ is finite. Then $F$ is $A C$ if and only if the functions $F_{r}, r=1, \cdots, m$, are all $A C$.

Proof. It is clear that $F$ satisfies condition (b) in the definition of $A C$ if and only if each $F_{r}$ also satisfies this condition. It remains to show that $F$ is additive if and only if each $F_{r}$ is additive. Let $I$ be an interval and $I=\cup_{j} I_{j}$ be a finite subdivision of $I$ into nonoverlapping intervals $I_{j}$. Let

$$
d=F(I)-\sum_{j} F\left(I_{j}\right), \quad d_{r}=F_{r}(I)-\sum_{j} F_{r}\left(I_{j}\right) .
$$

For each system $D=[J]$ let

$$
\begin{aligned}
d(D) & =\sum_{J} s(J, I)\left[1-\sum_{j} s\left(J, I_{j}\right)\right] F(J) \\
& =S[F, I, D]-\sum_{j} s\left[F, I_{j}, D\right], \\
d_{r}(D) & =\sum_{J} s(J, I)\left[1-\sum_{j} s\left(J, I_{j}\right)\right] F_{r}(J) \\
& =S\left[F_{r}, I, D\right]-\sum_{j} S\left[F_{r}, I_{j}, D\right] .
\end{aligned}
$$

As $\delta(D) \rightarrow 0$, we have $d(D) \rightarrow d$ and $d_{r}(D) \rightarrow d_{r}$ by (2.2). From the inequalities $F_{r}(J) \leqq F(J) \leqq \sum_{r} F_{r}(J)$ we obtain $d_{r}(D) \leqq d(D) \leqq \sum_{r} d_{r}(D)$. 
and, after a passage to the limit, $d_{r} \leqq d \leqq \sum_{r} d_{r}$. Since $F_{r}$ is overadditive and $d_{r} \geqq 0$, we conclude that $d=0$ if and only if $d_{r}=0$ for each $r$. This completes the proof.

Proposition 3.5. Suppose that $z$ is quasi additive on $A$ and that $\int[|z|, A]$ is finite. Then $D(w) \geqq|J(w)|$ a.e. in $A$ and equality holds if $F$ is $A C$.

The proof is essentially the same as given in parts (a) and (b) of the proof of $[1,30.1$ (ii)]: simply replace the letter $V$ by $F$ and the references to $[1,9.1]$ and $[1,12.1]$ by a reference to $(2.6)$ in the present paper.

Theorem 3.6. Suppose that $z$ is quasi additive on $A$ and that $\int[|z|, A]$ is finite. Then $F(A) \geqq \int_{A}|J(w)| d w$ and the same inequalities hold if $A$ is replaced by any open subset $G$ of $A$ or by any set $I \in\{I\}$. The equality $F(A)=\int_{A}|J(w)| d w$ holds if and only if $F$ is $A C$ and, in this case, the same equality holds if $A$ is replaced by any set $G$ or I as above.

This theorem is a consequence of (2.2), (3.1), and (3.5).

4. t-quasi additivity. We assume axioms (a)-(d) of $\S 1$ throughout this section. In addition, let there be associated with each subset $E$ of $A$ a set $E^{t}$ satisfying the condition

$\left(t_{1}\right): E^{t}$ is contained in the interior of $E$,

$\left(t_{2}\right): \quad E^{t} \subset G^{t}$ whenever $E \subset G \subset A$.

DeFINITION 4.1. $z$ is t-quasi additive on $M$ if, under the circumstances of Definition (1.1), $z$ satisfies

$\left(t q a_{1}\right): \quad \sum_{I} s(I, M)\left|\sum_{I} s\left(J, I^{t}\right) z(J)-z(I)\right|<\varepsilon$,

$\left(t q a_{2}\right): \quad \sum_{J} s(J, M)\left[1-\sum_{I} s\left(J, I^{t}\right) s(I, M)\right]|z(J)|<\varepsilon$.

An analogous definition of "t-quasi subadditivity" may be formulated if $z$ is real-valued. The statements of $\S 1$ remain valid if the terms "quasi additive", "quasi subadditive", and " $s(J, I)$ " are consistently replaced by " $t$-quasi additive", " $t$-quasi subadditive", and " $s\left(J, I^{t}\right)$ ", respectively. (We do not modify the definition of the $B-C$ integral.)

Proposition 4.2. If $z$ is $t$-quasi additive on $M$, then $z$ is also quasi additive on $M$. 
Proof. For any two systems $D_{0}=[I]$ and $D=[J]$ we have

$$
\begin{aligned}
\sum_{I} s & (I, M)\left[\sum_{J} s(J, I) z(J)-z(I)\right] \\
= & \sum_{I} s(I, M)\left[\sum_{J} s\left(J, I^{t}\right) z(J)-z(I)\right] \\
& \quad+\sum_{I} s(I, M) \sum_{J} s(J, I)\left[1-s\left(J, I^{t}\right)\right] z(J) .
\end{aligned}
$$

Let $\varepsilon>0$ be given and let $\eta=\eta(\varepsilon / 2, M)>0, D_{0}=[I]$ with $\delta\left(D_{0}\right)<\eta$, $\lambda=\lambda\left(\varepsilon / 2, M, D_{0}\right)>0$, and $D=[J]$ with $\delta(D)<\lambda$ be as in the definition of $t$-quasi additivity. Then

$$
\begin{gathered}
\sum_{I} s(I, M)\left|\sum_{J} s\left(J, I^{t}\right) z(J)-z(I)\right|<\varepsilon / 2, \\
\sum_{J} s(J, M)\left[1-\sum_{I} s\left(J, I^{t}\right) s(I, M)\right]|z(J)|<\varepsilon / 2 .
\end{gathered}
$$

The last term in (1) is less inclusive than the term in (3) and from (1)-(3) we obtain

$$
\sum_{I} s(I, M)\left|\sum_{J} s(J, I) z(J)-z(I)\right|<\varepsilon / 2+\varepsilon / 2=\varepsilon .
$$

The term in $(3)^{\prime}$ below is also less inclusive than the term in (3) and hence

$$
\sum_{J} s(J, M)\left[1-\sum_{I} s(J, I) s(I, M)\right]|z(J)|<\varepsilon / 2 .
$$

Relations $(2)^{\prime}$ and $(3)^{\prime}$ show that $z$ is quasi additive on $M$.

Proposition 4.3. Assume that each interval $I \in\{I\}$ is connected. If $z$ is t-quasi additive on $M$ and if $\int[|z|, M]$ is finite, then $F\left(M^{t}\right)=$ $F(M)$.

Proof. Let $\varepsilon>0$ be given and let $\mu=\mu(\varepsilon / 2, M), D_{0}=[I]$ with $\delta\left(D_{0}\right)<\mu, \lambda=\lambda\left(\varepsilon / 2, M, D_{0}\right)$, and $D=[J]$ with $\delta(D)<\lambda$ be as in the $t$-quasi additivity version of Proposition (1.4). Then

$$
\begin{aligned}
& \left|F(M)-S\left[|z|, M, D_{0}\right]\right|<\varepsilon / 2, \\
& |F(M)-S[|z|, M, D]|<\varepsilon / 2, \\
& \sum_{I} s(I, M)\left|\sum_{J} s\left(J, I^{t}\right)\right| z(J)|-| z(I)||<\varepsilon / 2 .
\end{aligned}
$$

Let $M^{\prime}$ denote the union of the sets $I^{t}$ such that $I \in D_{0}$ and $I \subset M$. Then $M^{\prime} \subset M^{t} \subset M$. Since each $I^{t}$ is contained in the interior of $I$ and since the intervals $J$ are connected, each interval $J \in D$ with $J \subset M^{\prime}$ is contained in $I^{t}$ for one and only one interval $I \in D_{0}$ with $I \subset M$. Hence 


$$
\sum_{J} s\left(J, M^{\prime}\right)|z(J)|=\sum_{I} s(I, M) \sum_{J} s\left(J, I^{t}\right)|z(J)|
$$

and therefore

$$
\begin{aligned}
\mid F(M) & -\sum_{J} s\left(J, M^{\prime}\right) \mid z(J) \| \\
\leqq & \left|F(M)-\sum_{I} s(I, M)\right| z(I)|| \\
& +\left|\sum_{J} s\left(J, M^{\prime}\right)\right| z(J)\left|-\sum_{I} s(I, M)\right| z(I)|| \\
& <\varepsilon / 2+\sum_{I} s(I, M)\left|\sum_{J} s\left(J, I^{t}\right)\right| z(J)|-| z(I)|| \\
& <\varepsilon / 2+\varepsilon / 2=\varepsilon .
\end{aligned}
$$

Thus, given $\varepsilon>0$, there is a set $M^{\prime}=M^{\prime}(\varepsilon), M^{\prime} \subset M^{t} \subset M$, and a number $\lambda=\lambda(\varepsilon)>0$ such that

$$
F(M)-\varepsilon<S\left[|z|, M^{\prime}, D\right] \leqq S\left[|z|, M^{t}, D\right] \leqq S[|z|, M, D]<F(M)+\varepsilon
$$

for every system $D$ with $\delta(D)<\lambda$. Thus, $F\left(M^{t}\right)=F(M)$.

Proposition 4.4. If $z$ is quasi additive on $M, \int[|z|, M]$ is finite, and $F\left(M^{t}\right)=F(M)$, then $\mathscr{F}\left(M^{t}\right)=\mathscr{F}(M)$ and similarly for $\mathscr{F}_{r}, F_{r}, F_{r}^{+}$, and $F_{r}^{-}, r=1, \cdots, m$.

Proof. We show that $\mathscr{F}\left(M^{t}\right)=\mathscr{F}(M)$; the other parts are proved in an analogous manner. All limits below are taken as $\delta(D) \rightarrow 0, D=[I]$. Since

$$
\begin{aligned}
F(M) & =\lim \sum_{I} s(I, M)|z(I)| \\
& =\lim \left\{\sum_{I} s\left(I, M^{t}\right)|z(I)|+\sum_{I} s(I, M)\left[1-s\left(I, M^{t}\right)\right]|z(I)|\right\}
\end{aligned}
$$

and

$$
F(M)=F\left(M^{t}\right)=\lim \sum_{I} s\left(I, M^{t}\right)|z(I)|<\infty,
$$

we conclude that

$$
\lim \sum_{I}(I, M)\left[1-s\left(I, M^{t}\right)\right]|z(I)|=0,
$$

and therefore

$$
\lim \sum_{I} s(I, M)\left[1-s\left(I, M^{t}\right)\right] z(I)=0
$$

Hence,

$$
\begin{aligned}
\mathscr{F}(M) & =\lim \left\{\sum_{I} s\left(I, M^{t}\right) z(I)+\sum_{I} s(I, M)\left[1-s\left(I, M^{t}\right)\right] z(I)\right\} \\
& =\mathscr{F}\left(M^{t}\right) .
\end{aligned}
$$


THEOREM 4.5. Assume that each interval $I \in\{I\}$ is connected, $z$ is quasi additive on $M$, and $\int[|z|, M]$ is finite. Then $z$ is t-quasi additive on $M$ if and only if $F\left(I^{t}\right)=F(I)$ for each interval $I \subset M$.

Proof. The condition is necessary by (4.3). Assume now that $F\left(I^{t}\right)=F(I)$ for each interval $I \subset M$. We must show that $z$ is $t$-quasi additive on $M$. Let $D_{0}=[I]$ and $D=[J]$ be any two systems. Then

$$
\begin{aligned}
& \sum_{I} s(I, M)\left|\sum_{J} s\left(J, I^{t}\right) z(J)-z(I)\right| \\
& \leqq\left\{\sum_{I} s(I, M)\left|\sum_{J} s\left(J, I^{t}\right) z(J)-\mathscr{F}(I)\right|\right\} \\
&+\left\{\sum_{I} s(I, M)\left|\mathscr{F}(I)-\sum_{J} s(J, I) z(J)\right|\right\} \\
&+\left\{\sum_{I} s(I, M)\left|\sum_{J} s(J, I) z(J)-z(I)\right|\right\} \\
&= s_{1}+s_{2}+s_{3},
\end{aligned}
$$

and

$$
\begin{aligned}
& \sum_{J} s(J, M)\left[1-\sum_{I} s\left(J, I^{t}\right) s(I, M)\right]|z(J)| \\
&= \sum_{J} s(J, M)|z(J)|-\sum_{J} \sum_{I} s\left(J, I^{t}\right) s(I, M)|z(J)| \\
& \leqq\left\{\left|\sum_{J} s(J, M)\right| z(J)\left|-\sum_{J} \sum_{I} s(J, I) s(I, M)\right| z(J) \|\right\} \\
&+\left\{\left|\sum_{J} \sum_{I} s(J, I) s(I, M)\right| z(J)\left|-\sum_{J} s(I, M) F(I)\right|\right\} \\
&+\left\{\left|\sum_{I} s(I, M) F(I)-\sum_{J} \sum_{I} s\left(J, I^{t}\right) s(I, M)\right| z(J) \|\right\} \\
&= s_{4}+s_{5}+s_{6} .
\end{aligned}
$$

Let $\varepsilon>0$ be given and let $\eta_{1}=\eta(z, \varepsilon / 3, M)$ and $\eta_{2}=\eta(|z|, \varepsilon / 3, M)$ be as in Definition (1.1). Let $\eta=\min \left[\eta_{1}, \eta_{2}\right]$ and let $D_{0}=[I]$ satisfy $\delta\left(D_{0}\right)<\eta$. Let $\lambda_{1}=\lambda\left(z, \varepsilon / 3, M, D_{0}\right)$ and $\lambda_{2}=\lambda\left(|z|, \varepsilon / 3, M, D_{0}\right)$ be as in Definition (1.1) and let $\lambda=\min \left[\lambda_{1}, \lambda_{2}\right]$. Let $N$ be the number of intervals $I \in D_{0}$ and let $\varepsilon^{\prime}=\varepsilon / N$. For each $I \in D_{0}$ with $I \subset M$ let $\mu_{I}=\mu\left(\varepsilon^{\prime} / 3, I\right)$ and $\mu_{I}^{t}=\mu\left(\varepsilon^{\prime} / 3, I^{t}\right)$ be as in (1.4). Let $\mu=\min$ $\left\{\lambda, \mu_{I}, \mu_{I}^{t}: I \in D_{0}, I \subset M\right\}$ and let $D=[J]$ satisfy $\delta(D)<\lambda$. Then
(4) $\left|\mathscr{F}\left(I^{t}\right)-S\left[z, I^{t}, D\right]\right|<\varepsilon^{\prime} / 3$ for each $I \in D_{0}$ with $I \subset M$,
(5) $|\mathscr{F}(I)-S[z, I, D]|<\varepsilon^{\prime} / 3$ for each $I \in D_{0}$ with $I \subset M$,
(6) $\sum_{I} s(I, M)\left|\sum_{J} s(J, I) z(J)-z(I)\right|<\varepsilon / 3$,
( 7 ) $\sum_{J} s(J, M)\left[1-\sum_{I} s(J, I) s(I, M)\right]|z(J)|<\varepsilon / 3$,
(8) $|F(I)-S[|z|, I, D]|<\varepsilon^{\prime} / 3$ for each $I \in D_{0}$ with $I \subset M$,
(9) $\left|F\left(I^{t}\right)-S\left[|z|, I^{t}, D\right]\right|<\varepsilon^{\prime} / 3$ for each $I \in D_{0}$ with $I \subset M$.

\section{Hence,}




$$
\begin{aligned}
0 \leqq s_{1}= & \sum_{I} s(I, M)\left|\sum_{J} s\left(J, I^{t}\right) z(J)-\mathscr{F}(I)\right| \\
= & \sum_{I} s(I, M)\left|\sum_{J} s\left(J, I^{t}\right) z(J)-\mathscr{F}\left(I^{t}\right)\right| \\
& <N\left(\varepsilon^{\prime} / 3\right)=\varepsilon / 3 \text { by }(4.4) \text { and }(4), \\
0 \leqq s_{2}= & \sum_{I} s(I, M)\left|\mathscr{F}(I)-\sum_{J} s(J, I) z(J)\right| \\
& <N\left(\varepsilon^{\prime} / 3\right)=\varepsilon / 3 \text { by }(5), \\
0 \leqq s_{3}= & \sum_{I} s(I, M)\left|\sum_{J} s(J, I) z(J)-z(I)\right|<\varepsilon / 3 \text { by }(6), \\
0 \leqq s_{4}= & \left|\sum_{J} s(J, M)\right| z(J)\left|-\sum_{J} \sum_{I} s(J, I) s(I, M)\right| z(J) \| \\
\leqq & \sum_{J} s(J, M)\left[1-\sum_{I} s(J, I) s(I, M)|z(J)|<\varepsilon / 3 \text { by }(7),\right. \\
0 \leqq s_{5}= & \left|\sum_{J} \sum_{I} s(J, I) s(I, M)\right| z(J)\left|-\sum_{I} s(I, M) F(I)\right| \\
\leqq & \sum_{I} s(I, M)\left|\sum_{J} s(J, I)\right| z(J)|-F(I)| \\
& <N\left(\varepsilon^{\prime} / 3\right)=\varepsilon / 3 \text { by }(8), \\
0 \leqq s_{6}= & \left|\sum_{I} s(I, M) F(I)-\sum_{J} \sum_{I} s\left(J, I^{t}\right) s(I, M)\right| z(J) \| \\
\leqq & \sum_{I} s(I, M)\left|F(I)-\sum_{J} s\left(J, I^{t}\right)\right| z(J) \| \\
= & \sum_{I} s(I, M)\left|F\left(I^{t}\right)-\sum_{J} s\left(J, I^{t}\right)\right| z(J) \| \\
< & N\left(\varepsilon^{\prime} / 3\right)=\varepsilon / 3 \text { by }(9)
\end{aligned}
$$

Thus $s_{1}+s_{2}+s_{3}<\varepsilon, s_{4}+s_{5}+s_{6}<\varepsilon$, and we conclude that $z$ is $t$-quasi additive on $M$.

We remark that the connectedness of the intervals $I \in\{I\}$ is not used in the sufficiency part of the above proof.

5. Remarks. Definition (1.1) was used under axioms (a)-(d) by Cesari [2] for the case $M=A$. In [3] Cesari extended the notions of B-C integral and quasi additivity to a class $\{G\}$ of nonempty subsets of $A$ as follows. For each $G$ in $\{G\}$ let $\{D\}_{G}$ be the family of all systems $D_{G}=\{I \in D: I \subset G\}$ obtained as $D$ ranges over the family $\{D\}$, and let $\delta_{G}$ be a mesh satisfying axiom (d) relative to $\{D\}_{G}$. In addition, assume the axiom

(e): given $\tau>0$, there exists $\nu=\nu(\tau, G)>0$ such that if $D$ is any system in $\{D\}$ with $\delta(D)<\nu$, then the associated system $D_{G}=\{I \in D: I \subset G\}$ is nonempty and $\delta_{G}\left(D_{G}\right)<\tau$.

Cesari then defined B-C integrals over $G$ and quasi additivity on $G$ relative to the directed system $\left(\{D\}_{G}, \delta_{G}\right)$; axiom (e) was used to obtain properties of the $\mathrm{B}-\mathrm{C}$ integrals as set functions.

To see that Cesari's formulation is contained in that of the present paper we observe the following two statements. 
(i ) $\lim _{\delta(D) \rightarrow 0} S[z, G, D]=\lim _{\delta_{G}\left(D_{G}\right) \rightarrow 0} S\left[z, G, D_{G}\right]$ whenever axiom (e) holds and the limit on the right exists.

(ii) If $z$ is quasi additive [subadditive] on $G$ relative to $\left(\{D\}_{G}, \delta_{G}\right)$ and if axiom (e) holds, then $z$ is quasi additive [subadditive] on $G$ relative to $(\{D\}, \delta)$.

Statement (i) was proved by Cesari [3, p. 117], and (ii) may be proved in an analogous manner. Since simple examples show that if $\int[|z|, G]=0$ there may no mesh $\delta_{G}$ satisfying axiom (e), some improvement is gained by formulating all quasi additivity relations relative to the single directed system $(\{D\}, \delta)$ as in the present paper. The theorems proved by Cesari [3] carry over to the present setting, moreover, with only the obvious changes in the mesh conditions required. For the sake of completeness, we shall next restate the most important of these theorems.

Let $\mathscr{T}$ denote the topology on $A, \mathscr{G}$ be a topology on $A$ coarser than $\mathscr{F}$, and $\mathscr{B}$ be the $\sigma$-algebra on $A$ generated by $\mathscr{C}$. In addition to the axioms (a)-(d) of $\S 1$, assume the following four additional hypotheses.

$\left(H_{1}\right): \quad z$ is quasi additive on $A$ and $\int[|z|, A]$ is finite.

$\left(H_{2}\right)$ : Each interval $I \in\{I\}$ is $\mathscr{T}$-connected.

$\left(H_{3}\right)$ : If $G=\cup_{n} G_{n}$ is a countable union of sets $G_{n} \in \mathscr{G}$, then $F(G) \leqq \sum_{n} F\left(G_{n}\right)$, and analogously for $F_{r}, F_{r}^{+}$, and $F_{r}^{-}, r=1, \cdots, m$.

$\left(H_{4}\right):$ If $G \in \mathscr{G}$, then $F(G)=\sup F\left(G^{\prime}\right)$ where the supremum is taken over all sets $G^{\prime} \in \mathscr{G}$ whose $\mathscr{G}$-closure is contained in $G$, and analogously for $F_{r}, F_{r}^{+}$, and $F_{r}^{-}, r=1, \cdots, m$.

Neither $\left(H_{3}\right)$ nor $\left(H_{4}\right)$ is a consequence of the axioms or preceding hypotheses. Finally, for each subset $M$ of $A$, define

$$
\begin{aligned}
\mu(M) & =\inf F(G), & \mu_{r}(M) & =\inf F_{r}(G), \\
\mu_{r}^{-}(M) & =\inf F_{r}^{+}(G), & \mu_{r}^{-}(M) & =\inf F_{r}^{-}(G), \\
\nu_{r}(M) & =\mu_{r}^{+}(M)-\mu_{r}^{-}(M), & \nu(M) & =\left(\nu_{1}(M), \cdots, \nu_{m}(M)\right),
\end{aligned}
$$

where the infima are taken over all sets $G \in \mathscr{C}$ with $M \subset G$.

With the help of $\left(H_{1}\right)$ and Theorem (1.4), we see that

$$
\begin{aligned}
& \mu_{r}(M)=\mu_{r}^{+}(M)+\mu_{r}^{-}(M), \\
& \left|\nu_{r}(M)\right| \leqq \mu_{r}(M) \leqq \mu(M), \\
& |\nu(M)| \leqq\left[\sum_{r} \mu_{r}^{2}(M)\right]^{1 / 2} \leqq \mu(M) \leqq \sum_{r} \mu_{r}(M),
\end{aligned}
$$

for each $r$ and $M$. Moreover, the set functions $\mu, \mu_{r}, \mu_{r}^{+}, \mu_{r}^{-}, \nu_{r}$, and $\nu$ agree on $\mathscr{G}$ with the set functions $F, F_{r}, F_{r}^{+}, F_{r}^{-}, \mathscr{F}_{r}$, and $\mathscr{F}$, respectively. 
Proposition 5.1. Under hypotheses $\left(H_{1}\right)-\left(H_{4}\right)$, the set functions $\mu, \mu_{r}, \mu_{r}^{+}$, and $\mu_{r}^{-}, r=1, \cdots, m$, are outer measures on $A$ and are finite measures on $\mathscr{B}$.

It follows that the set functions $\nu_{r}$ are signed measures on $\mathscr{B}$ which are absolutely continuous with respect to the measure $\mu$. Thus we may define the Radon-Nikodym derivatives

$$
\theta_{r}=d \nu_{r} / d \mu, \quad \theta=\left(\theta_{1}, \cdots, \theta_{m}\right) .
$$

As Cesari observed, the relations $\nu_{r}=\mu_{r}^{+}-\mu_{r}^{-}$need not represent Jordan decompositions of the signed measures $\nu_{r}$. This situation is rectified by replacing $\left(H_{1}\right)$ by the slightly stronger hypothesis

$\left(H_{1}\right)^{\prime}: \quad z$ is $t$-quasi additive on $A$ and $\int[|z|, A]$ is finite, where $t$ denotes the interior operator for the topology $\mathscr{C}$.

By (4.5), $\left(H_{1}\right)^{\prime}$ and $\left(H_{2}\right)$ are equivalent to $\left(H_{1}\right),\left(H_{2}\right)$, and the statement that $F(I)=F\left(I^{t}\right)$ for every interval $I$.

Proposition 5.2. Assume $\left(H_{1}\right)^{\prime}$ and $\left(H_{2}\right)-\left(H_{4}\right)$. Then

(i) $\nu_{r}=\mu_{r}^{+}-\mu_{r}^{-}$represent Jordan decompositions,

(ii) $\mu(B)=\sup \sum_{j}\left[\sum_{r} \mu_{r}^{2}\left(B_{j}\right)\right]^{1 / 2}=\sup \sum_{j}\left|\nu\left(B_{j}\right)\right|$, where $B$ is an arbitrary set in $\mathscr{B}$ and the suprema are taken over all finite decompositions $B=\cup_{j} B_{j}$ of $B$ into sets $B_{j}$ in $\mathscr{B}$,

(iii) $|\theta|=1 \mu-$ a.e. in $A$.

We turn now to Cesari's theorem on the existence and representation of Cesari-Weierstrass integrals. Let $T: A \rightarrow K, x=T(w)$, be a mapping from $A$ into a metric space $K$, and let $f: K \times E_{m} \rightarrow E_{1}$, $a=f(x, q)$, be a real-valued function defined on the product space $K \times E_{m}$. Let $S^{m-1}=\left\{q \in E_{m}:|q|=1\right\}$ be the unit sphere in $E_{m}$. Finally, let $w_{I}$ denote an arbitrary point of $I$ for each interval $I \in\{I\}$.

Theorem 5.3. Suppose

(i) $\max \{$ diameter $T(I): I \in D\} \leqq \delta(D)$ for each $D \in\{D\}$,

(ii) $f$ is bounded and uniformly continuous on $K \times S^{m-1}$,

(iii) $f(x, t q)=t f(x, q)$ for all $t \geqq 0, x \in K$, and $q \in E_{m}$. Then, under hypothesis $\left(H_{1}\right)$, the real-valued interval function

$$
Z(I)=f\left[T\left(w_{I}\right) z(I)\right]
$$

is quasi additive on $A$, the parameters of Definition (1.1) can be determined independently of the choice of $w_{I} \in I$, and the value of the $B-C$ integral

$$
\int[f(T, z), A]=\int[Z, A]
$$


is independent of the choice of $w_{I} \in I$. Further, under the hypotheses $\left(H_{1}\right)^{\prime}$ and $\left(H_{2}\right)-\left(H_{4}\right)$, the function $f[T(w), \theta(w)], w \in A$, is $\mu$-integrable on $A$ and

$$
\int[f(T, z), A]=\int_{A} f[T(w), \theta(w)] d \mu
$$

The proof, given in [2,3] for $K$ a subset of some Euclidean space, is valid if $K$ is simply a metric space.

\section{REFERENCES}

1. L. Cesari, Surface Area, Princeton University Press (1956).

2. - Quasi-additive set functions and the concept of integral over a variety, Trans. Amer. Math. Soc., 102 (1962), 94-113.

3. - Extension problem for quasi-additive set functions and Radon-Nikodym derivatives, Trans. Amer. Math. Soc., 102 (1962), 114-146.

4. T. Nishiura, Integrals over a product variety and Fubini theorems, Rend. Circ. Mat. Palermo, 14 (1965), 207-236.

5. T. Rado and P. V. Reichelderfer, Continuous Transformations in Analysis, Springer-Verlag, Berlin (1955).

6. A. Stoddart, Integrals of the calculus of variations, Thesis, University of Michigan (1964).

7. G. Warner, Quasi additive set functions and non-linear integration over a variety, Thesis, University of Michigan (1966).

8. — The Burkill-Cesari integral, Duke Math. J., 35 (1968), 61-78.

9. —— The generalized Weierstrass-type integral $\int f(\zeta, \varphi)$, Ann. Scuola Norm. Sup. Pisa (2), 22 (1968), 163-192.

Received September 1, 1970. A portion of this paper was taken from the author's doctoral dissertation written under the direction of Professor L. Cesari at the University of Michigan, 1969. (AFOSR Project 69-1662.)

WAYNe State University 


\title{
PACIFIC JOURNAL OF MATHEMATICS
}

\author{
EDITORS
}

\author{
H. SAMelson \\ Stanford University \\ Stanford, California 94305 \\ C. R. HoBBy \\ University of Washington \\ Seattle, Washington 98105
}

J. DugunduI

Department of Mathematics

University of Southern California

Los Angeles, California 90007

RICHARD ARENS

University of California

Los Angeles, California 90024

\section{ASSOCIATE EDITORS}

\author{
E. F. BeCKENBACH
}

B. H. NEUMANN

F. WoLF

K. YOSHIDA

\section{SUPPORTING INSTITUTIONS}

\author{
UNIVERSITY OF BRITISH COLUMBIA \\ CALIFORNIA INSTITUTE OF TECHNOLOGY \\ UNIVERSITY OF CALIFORNIA \\ MONTANA STATE UNIVERSITY \\ UNIVERSITY OF NEVADA \\ NEW MEXICO STATE UNIVERSITY \\ OREGON STATE UNIVERSITY \\ UNIVERSITY OF OREGON \\ OSAKA UNIVERSITY \\ UNIVERSITY OF SOUTHERN CALIFORNIA
}

\author{
STANFORD UNIVERSITY \\ UNIVERSITY OF TOKYO \\ UNIVERSITY OF UTAH \\ WASHINGTON STATE UNIVERSITY \\ UNIVERSITY OF WASHINGTON \\ AMERICAN MATHEMATICAL SOCIETY \\ CHEVRON RESEARCH CORPORATION \\ NAVAL WEAPONS CENTER
}

The Supporting Institutions listed above contribute to the cost of publication of this Journal, but they are not owners or publishers and have no responsibility for its content or policies.

Mathematical papers intended for publication in the Pacific Journal of Mathematics should be in typed form or offset-reproduced, (not dittoed), double spaced with large margins. Underline Greek letters in red, German in green, and script in blue. The first paragraph or two must be capable of being used separately as a synopsis of the entire paper. The editorial "we" must not be used in the synopsis, and items of the bibliography should not be cited there unless absolutely necessary, in which case they must be identified by author and Journal, rather than by item number. Manuscripts, in duplicate if possible, may be sent to any one of the four editors. Please classify according to the scheme of Math. Rev. Index to Vol. 39. All other communications to the editors should be addressed to the managing editor, Richard Arens, University of California, Los Angeles, California, 90024.

50 reprints are provided free for each article; additional copies may be obtained at cost in multiples of 50 .

The Pacific Journal of Mathematics is published monthly. Effective with Volume 16 the price per volume (3 numbers) is $\$ 8.00$; single issues, $\$ 3.00$. Special price for current issues to individual faculty members of supporting institutions and to individual members of the American Mathematical Society: $\$ 4.00$ per volume; single issues $\$ 1.50$. Back numbers are available.

Subscriptions, orders for back numbers, and changes of address should be sent to Pacific Journal of Mathematics, 103 Highland Boulevard, Berkeley, California, 94708.

PUBLISHED BY PACIFIC JOURNAL OF MATHEMATICS, A NON-PROFIT CORPORATION

Printed at Kokusai Bunken Insatsusha (International Academic Printing Co., Ltd.), 7-17, Fujimi 2-chome, Chiyoda-ku, Tokyo, Japan. 


\section{Pacific Journal of Mathematics}

\section{Vol. 37, No. $3 \quad$ March, 1971}

Mohammad Shafqat Ali and Marvin David Marcus, On the degree of the

minimal polynomial of a commutator operator ................ 561

Howard Anton and William J. Pervin, Integration on topological

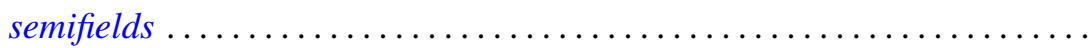

Martin Bartelt, Multipliers and operator algebras on bounded analytic

functions .................................... 575

Donald Earl Bennett, Aposyndetic properties of unicoherent continua ...... 585

James W. Bond, Lie algebras of genus one and genus two ............. 591

Mario Borelli, The cohomology of divisorial varieties ............... 617

Carlos R. Borges, How to recognize homeomorphisms and isometries ....... 625

J. C. Breckenridge, Burkill-Cesari integrals of quasi additive interval

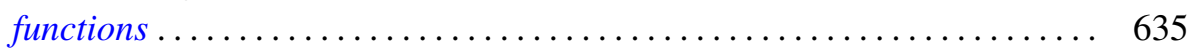

J. Csima, A class of counterexamples on permanents ................ 655

Carl Hanson Fitzgerald, Conformal mappings onto $\omega$-swirly domains . . . . . . 657

Newcomb Greenleaf, Analytic sheaves on Klein surfaces .............. 671

G. Goss and Giovanni Viglino, C-compact and functionally compact

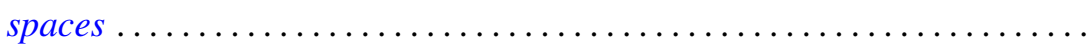

Charles Lemuel Hagopian, Arcwise connectivity of semi-aposyndetic plane

continua ..................................... 683

John Harris and Olga Higgins, Prime generators with parabolic limits ...

David Michael Henry, Stratifiable spaces, semi-stratifiable spaces, and their

relation through mappings .......................

Raymond D. Holmes, On contractive semigroups of mappings ........... 701

Joseph Edmund Kist and P. H. Maserick, BV-functions on semilattices ....... 711

Shûichirô Maeda, On point-free parallelism and Wilcox lattices ........... 725

Gary L. Musser, Linear semiprime $(p ; q)$ radicals ................. 749

William Charles Nemitz and Thomas Paul Whaley, Varieties of implicative

semilattices..................................... 759

Jaroslav Nešetřil, A congruence theorem for asymmetric trees ............ 771

Robert Anthony Nowlan, A study of $H$-spaces via left translations .......... 779

Gert Kjærgaard Pedersen, Atomic and diffuse functionals on a $C^{*}$-algebra ... 795

Tilak Raj Prabhakar, On the other set of the biorthogonal polynomials

suggested by the Laguerre polynomials...

801

Leland Edward Rogers, Mutually aposyndetic products of chainable

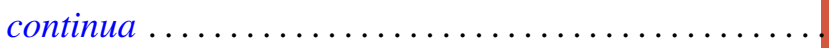

Frederick Stern, An estimate for Wiener integrals connected with squared

error in a Fourier series approximation.

Leonard Paul Sternbach, On k-shrinking and k-boundedly complete basic

sequences and quasi-reflexive spaces .................... 817

Pak-Ken Wong, Modular annihilator $A^{*}$-algebras ........ 